\title{
Cross-sectoral video consultation in cancer care: GPs' evaluation of a randomised controlled trial
}

\section{Theis Bitz Trabjerg ${ }^{1 *}$, Lars Henrik Jensen ${ }^{2,3}$, Jens Sondergaard ${ }^{1}$, Sonja Wehberg ${ }^{1}$, Jeffrey James Sisler ${ }^{4}$, Dorte Gilså Hansen ${ }^{1,5}$}

${ }^{1}$ Research Unit of General Practice, Department of Public Health, University of Southern Denmark, Odense, Denmark; ${ }^{2}$ Department of Oncology, Lillebælt Hospital, University Hospital of Southern Denmark, Vejle, Denmark; ${ }^{3}$ Department of Regional Health Research, Lillebælt Hospital, Center of Clinical Excellence, Danish Colorectal Cancer Center South, University Hospital of Southern Denmark, Vejle, Denmark; ${ }^{4}$ Department of Family Medicine, Faculty of Health Sciences, University of Manitoba, Winnipeg, MB, Canada; ${ }^{5}$ Center for Shared Decision Making, Lillebælt Hospital, University Hospital of Southern Denmark, Vejle, Denmark

\begin{abstract}
Background: Shared care models present an opportunity for patients to receive the benefits of specialist care combined with the continuity of care provided by a GP.

Aim: To test the effects on GP-perceived involvement in cancer care and their satisfaction with this cross-sectoral information after bringing the patient, GP, and oncologist together in a shared video consultation.
\end{abstract}

Design \& setting: GPs from the Region of Southern Denmark evaluated a randomised controlled trial testing shared video consultations.

Method: This study describes secondary outcomes based on a 4 months' follow-up survey from GPs participating in The Partnership Project (PSP). Patient perception of coordination of care at 7 months' follow-up was the primary outcome of the PSP. A tripartite video consultation was conducted during cancer treatment to share tasks and roles between health professionals with the patient.

Results: The study included 281 patients, and 105 unique GPs returned 124 questionnaires. Video consultations were accomplished in $68 \%$ of scheduled cases. The study found an increased odds ratio (OR) of 3.03 for GP satisfaction with the distribution of tasks and roles, and they experienced more involvement in the cancer patients' trajectory. The study found an increased OR of 6.95 for the GP perception of more direct contact and dialogue with the Department of Oncology. There was a decreased OR of 0.88 for the GP to be engaged in handling anxiety and psychological concerns.

Conclusion: The study showed that involving the GP in one shared consultation increased the odds of the GP being satisfied with the distribution of tasks and roles, and feeling more involved in the cancer patient's trajectory. However, recruitment and response rates from GPs were limiting factors.

\section{How this fits in}

Shared video consultation between cancer patient, oncologist, and GP may increase the GP's perception of information exchange, involvement in cancer care, and clarification of tasks and roles between sectors. This evaluation of secondary outcomes from a randomised controlled trial showed that a 15-minute shared video consultation can increase the GPs' satisfaction and clarification of their 
task and roles during a cancer trajectory. Future patient-reported outcomes will investigate if this intervention also benefits patients.

\section{Introduction}

More involvement of primary care is suggested to improve the quality of cancer care. Giving the best support during and following long-term cancer treatment poses considerable challenges for GPs. ${ }^{1-4}$ GPs request better communication and collaboration with hospital specialists. ${ }^{5}$ Enhanced involvement by the GP is demanded by cancer patients. ${ }^{6-8}$

To provide effective cancer care, new patient-centred models supporting the exchange of knowledge and task clarification between oncologists and GPs are needed. ${ }^{9}$ Shared care is understood as 'an organizational model involving both primary care physicians and specialists in a formal, explicit manner'. ${ }^{10}$ Different elements are thought to improve treatment quality, like formal communication channels, role clarification, and enhanced patient confidence in GPs skills and competences. Shared care models present an opportunity for patients to receive the benefits of specialist care combined with the continuity of care and management of comorbidity provided by the GP. ${ }^{11}$ This implies a patient-centred outset and the development of a supporting communication model. ${ }^{12}$ Bringing both patient, GP, and oncologist together in a shared consultation might be a powerful solution as an add-on to usual care, consisting of written information between sectors, without involving the cancer patient.

Due to geographical reasons and shortage of health professionals' time, shared consultations are not feasible in daily routine. Video consultations have become increasingly common, ${ }^{13,14}$ and videobased communication may be an alternative solution to connect health professionals. ${ }^{15-17}$ The authors were not aware of studies exploring video consultations bringing a patient together with their GP and oncologist. However, video solutions have been used for multidisciplinary team meetings in cancer treatment planning. ${ }^{18}$ Recent trials have included GPs ${ }^{19}$ or patients, ${ }^{20,21}$ but not simultaneously. Therefore, the authors developed the partnership shared care model, including multidisciplinary video consultations between a cancer patient, oncologist, and GP. ${ }^{22}$ Based on a randomised controlled trial, this study aimed to test the effects on GP perceived involvement in cancer care, and their satisfaction with cross-sectoral information and coordination of care.

\section{Method}

\section{Study design}

This study reported secondary outcomes based on a 4 months' follow-up survey from GPs participating in the randomised controlled trial, PSP. ${ }^{22}$ Inclusion of patients was accomplished between June 2016 and November 2019. Patient perception of coordination of care at 7 months' follow-up was the primary outcome. ${ }^{22}$ This study follows the CONSORT statement ${ }^{23}$ (see Supplementary Table S1).

\section{Setting}

Patients were invited to participate in the study at the Department of Oncology, Lillebælt Hospital, if aged $>18$ years, treated with chemotherapy for a newly diagnosed cancer, and listed with a GP in the Region of Southern Denmark. GPs were all specialists in family medicine. ${ }^{22}$

Around 1300 cancer patients are referred to chemotherapy at the Department of Oncology, annually. The Region has 500 GPs working in approximately 300 general practices, comprised of 1 to 8 GPs per practice.

\section{The Partnership Project intervention}

The PSP consultation was planned as early as possible within 12 weeks from time of inclusion, corresponding to a maximum of 18 weeks after the first appointment at the department. The consultation was conducted as part of the planned standard programme at the hospital. If the patient chose to be located at the GP's office, a further consultation was scheduled. Consultations were booked 3 to 6 weeks in advance within regular clinic hours.

Before each consultation, oncologists and GPs received specific information about the aim of the consultation, including a consultation guide (Box 1) with themes that may be relevant. Not all 
Box 1 The consultation guide to GPs and oncologists, including themes potentially relevant for the consultation

Consultation guide:

- The oncologist acts as chair of the shared video consultation

- The duration of the consultation should be between 10 to 20 minutes

- The oncologist starts by introducing the participants and the purpose of the shared consultation

- Exchange of information between all participants for the benefit of the patient

- Role and tasks clarification between the Department of Oncology and the GP

- The consultations conclude with a summary, in which it is clarified whether a follow-up is needed with the GP or Department of Oncology

- The consultation and its agreements are documented in the hospitals' electronic patient record and sent to the GP, and made available for the patient online

The list of potential themes (not all themes might be relevant for the patient):

- A summary of the patient trajectory

- Patients concerns and desire for the consultation

- Sharing knowledge regarding comorbidity

- Psychosocial resources and needs

- Agreements on who should take care of what and when in the future

- Physical and psychological well-being

- Medicine

- Relatives

- Ability to work

- Late complications and side effects to the treatment

themes were raised in each consultation, but all themes were found relevant and discussed in different consultations. ${ }^{24}$

PSP consultations were accomplished through a video link using a virtual meeting room.

The control group received 'usual care', which included an electronic summary letter to the GP after each visit to the Department of Oncology. In case of questions, GPs could always telephone the hospital, and vice versa. Furthermore, patients could freely contact their GP or a specific nurse coordinator at the Department of Oncology.

\section{Randomisation and blinding}

After informed consent and a baseline questionnaire, patients were allocated in a 1:1 ratio using block randomisation. The GPs and oncologists could have patients in both groups.

Neither patients, GPs, nor oncologists in the intervention group were blinded to the patient's allocation. During enrolment and baseline data, patients and enrolling nurses were not aware of the randomisation. Data analysts were kept blinded. GPs to patients in the control arm were not formally informed before receiving the survey.

\section{Outcome measures}

The authors developed a GP questionnaire, ${ }^{22}$ including the number of patient visits to the GP and 28 items covering five different themes: GP assessment of the contact between the hospital and GP (two items), information from the hospital to the GP (seven items), GP involvement in the trajectory (six items), information from the hospital to help the GP (six items), and GP satisfaction with the distribution of task and roles (seven items) (see Supplementary Table S2). All questionnaires were measured on a 4-point Likert scale and used the following 'not at all', 'little', 'partly', and 'a lot', or, for linguistic reasons, 'very insufficient', 'partly insufficient', 'satisfactory', and 'very satisfactory'. For analysis, answers were dichotomised with two in favour of the intervention, and two in favour of usual care. Finally, GPs were given the option of commenting (data not shown).

\section{Data collection}

For data availability reasons, GPs in the control group were sent the questionnaire by postal service. The intervention group were managed using RedCap ${ }^{\circledR},{ }^{25}$ which automatically emailed secured links with the surveys. Due to Danish data protection laws, it was not possible to retrain demographic information on GPs who declined to participate or were non-responders. 


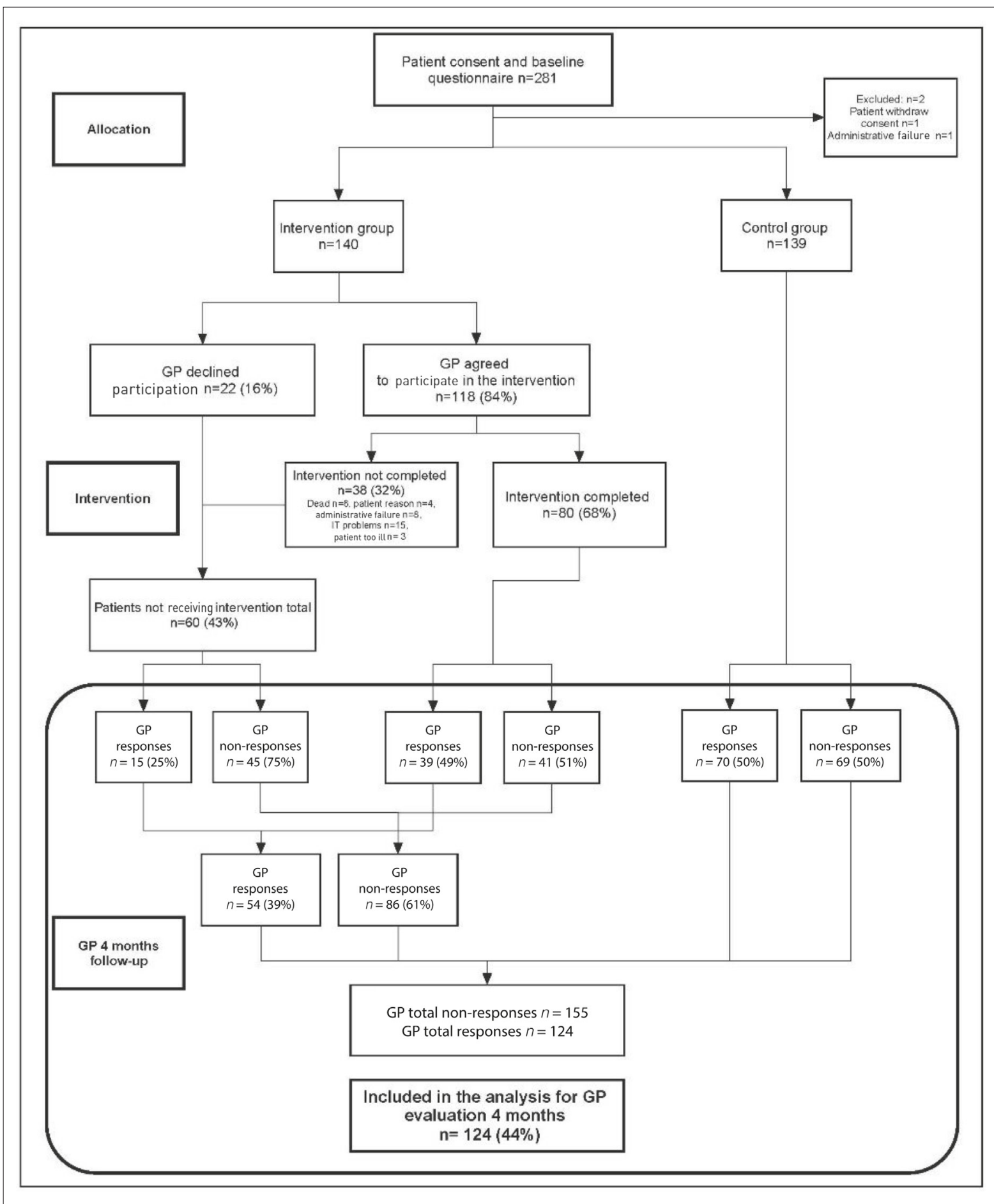

Figure 1 Study flow. 


\section{Sample size}

The number of GPs invited was based on sample size calculations, expressly the primary, patientreported outcome..$^{22}$ The goal was to include 278 patients (Figure 1).

\section{Statistical analysis}

Before performing the between-group analysis, six of the 29 items were chosen as outcomes. The selection was based on the process evaluations of the $\mathrm{PSP}_{1}{ }^{24,26}$ the overall distribution of items with an observed floor and ceiling effect for some items, and comments from the survey (data not shown).

For the between-groups analysis, a sensitivity analysis was performed with intention-to-treat and per-protocol analysis. GPs in the intervention group who participated in a video consultation as intended (with video and sound) were defined as having completed per-protocol.

The authors estimated the effect of group status in univariable logistic regression models, taking clustering by GP into account, and presented the results as OR.

Missing values were not imputed. Corrections for multiple testing were not employed. Due to the relatively small sample size, the authors chose not to fit multivariable models.

A $P$-value $<0.05$ was considered statistically significant.

\section{Results}

Demographic data on patients and GPs are shown in Tables 1 and 2.

In total, 281 patients were included. A total of 124 (44\%) questionnaires were returned from 105 unique GPs, eight having patients in both groups and nine GPs paticipated with $>1$ patient. (Figure 1).

A total of 80 (68\%) video consultations were completed as planned (Figure 1). For these, $64(80 \%)$ patients were located at the Department of Oncology, 16 (20\%) by the GP. A total of 25 unique oncology specialists had 1 to 13 consultations each.

The number of patient visits to general practice during the 4 months' follow-up was higher among patients in the intervention group than in the control group (data not shown).

GPs' answers are shown as frequencies in Supplementary Table S2. The regression analysis showed statistically significant OR in favour of the intervention regarding the item 'As a GP, did you experience direct contact or dialogue with the Department of Oncology?' (OR 6.95, 95\% confidence interval [CI] $=2.96$ to 16.35 ) and the item 'As a GP, how satisfied are you, with the distribution of tasks and roles regarding initiatives practice could initiate concerning the patient trajectory?' (OR $3.03,95 \% \mathrm{Cl}=1.35$ to 6.82). OR was significant and in the same direction for both intention-to-treat and per-protocol analysis. Regarding the item 'As a GP, I have been involved in handling the patient's anxiety and psychological concerns', the study found statistically significant decreased odds $(\mathrm{OR} 0.44,95 \% \mathrm{Cl}=$ 0.21 to 0.92$)$. The per-protocol analysis resulted in an OR in the same direction, but was insignificant (Table 3).

Two items showed insignificantly increased OR: 'As a GP, I have been involved in the treatment of comorbidity' and 'As a GP, how satisfied are you, with the distribution of tasks and roles of who should take care of side-effects and late complications?'; while insignificantly decreased OR was observed for 'As a GP, I found the information from the department helpful in managing the physical issues' (Table 3).

\section{Discussion}

\section{Summary}

The evaluation of the PSP randomised controlled trial showed that involving the GP in one consultation with the cancer patient and oncologist could significantly enhance the OR for the GPs being satisfied with the distribution of tasks and roles between sectors, and feeling well informed regarding how to support the cancer patient. This new way of shared care was compared with usual care. These results also suggested less involvement of GPs in handling the patients' anxiety and psychological problems. Recruitment and response rates from the GPs were challenging, with a lower than expected total response rate of $44 \%$, which should be considered when interpreting the results.

The result suggesting a mechanism of decreased involvement in anxiety and psychological issues by the GP did not support the authors' hypothesis. The decrease could either be an artefact, or perhaps 
Table 1 Baseline patient characteristic of the total patient population and the groups based on 105 unique GPs' responses represented by GP responses and non-responses, respectively

\begin{tabular}{|c|c|c|c|}
\hline Patient characteristic & All $(n=279), n(\%)$ & $\begin{array}{l}\text { GP non-responses } \\
(n=154), n(\%)\end{array}$ & $\begin{array}{c}\text { GP responses }(n=124) \text {, } \\
n(\%)\end{array}$ \\
\hline Mean age, years (SD) & $65.3(10.6)$ & $65.7(10.8)$ & $64.8(10.3)$ \\
\hline \multicolumn{4}{|l|}{ Sex } \\
\hline Male & $155(55.6)$ & $90(58.1)$ & $65(52.4)$ \\
\hline Female & $124(44.4)$ & 65 (41.9) & $59(47.6)$ \\
\hline \multicolumn{4}{|l|}{ Education } \\
\hline Primary school & $161(57.7)$ & $86(55.8)$ & $75(60.5)$ \\
\hline Upper secondary school & $16(5.7)$ & $10(6.5)$ & $6(4.8)$ \\
\hline Further education & $76(27.2)$ & $48(31.0)$ & $28(22.6)$ \\
\hline Higher education & $16(5.7)$ & $6(3.9)$ & $10(8.1)$ \\
\hline Missing & $10(3.6)$ & $4(32.6)$ & $5(4.0)$ \\
\hline \multicolumn{4}{|l|}{ Marital status } \\
\hline Single & $80(28.7)$ & $46(29.7)$ & $34(27.4)$ \\
\hline $\begin{array}{l}\text { Married/living with } \\
\text { someone }\end{array}$ & $198(71.0)$ & $108(69.7)$ & $90(72.6)$ \\
\hline \multicolumn{4}{|l|}{ Children at home } \\
\hline No & $244(87.5)$ & $134(86.5)$ & $110(88.7)$ \\
\hline Yes & $34(12.2)$ & 20 (12.9) & $14(11.3)$ \\
\hline \multicolumn{4}{|l|}{ Work status } \\
\hline Employed & 89 (31.9) & $50(32.3)$ & $39(31.5)$ \\
\hline Public benefits & $15(5.4)$ & $9(5.8)$ & $6(4.8)$ \\
\hline Retired & $174(62.4)$ & $95(61.3)$ & 79 (63.7) \\
\hline \multicolumn{4}{|l|}{ Comorbidity } \\
\hline No & $132(47.3)$ & 80 (51.9) & $51(41.1)$ \\
\hline Yes & $147(52.7)$ & 74 (47.7) & $73(58.9)$ \\
\hline \multicolumn{4}{|l|}{ Diagnosis/cancer type } \\
\hline Breast & $33(11.8)$ & $17(11.0)$ & $16(12.9)$ \\
\hline Gynaecological & $13(4.7)$ & $6(3.9)$ & $7(5.6)$ \\
\hline Lung & $106(38.0)$ & $64(41.6)$ & 42 (33.9) \\
\hline Gastrointestinal & $111(39.8)$ & $58(37.7)$ & $53(42.7)$ \\
\hline Other & $16(5.7)$ & $9(5.8)$ & $6(4.8)$ \\
\hline
\end{tabular}

Percentages do not come to $100 \%$ in all categories due to missing data. However, there a too few missing data to report due to data security.

patients seeing both doctors communicating gave them more peace of mind, thereby reducing the anxiety and leading to less involvement from the GP.

\section{Strengths and limitations}

The current study has several limitations to be considered. Sample bias due to a challenging recruitment process of patients, and subsequently their GPs, lead to the low overall response rate. Even though GPs are often challenging to recruit for research studies, ${ }^{27-29}$ the response rate in this study was considered lower than expected. ${ }^{30}$ These numbers, and the fact that GPs with a greater interest in cancer patients may be more likely to respond, might give room for selection bias. Due 
Table 2 Baseline characteristic of GPs participating in the survey (responders)

\begin{tabular}{lccc}
\hline GP variables & All $(n=124), n(\%)$ & $\begin{array}{c}\text { Intervention group } \\
(n=56), n(\%)\end{array}$ & $\begin{array}{c}\text { Control group }(n=68), \\
n(\%)\end{array}$ \\
\hline Sex & $61(49.2)$ & $29(51.8)$ & $32(47.1)$ \\
\hline Male & $63(50.8)$ & $27(48.2)$ & $36(52.9)$ \\
\hline Female & & & $24(35.3)$ \\
\hline Practicing family medicine, years & $43(34.7)$ & $19(33.9)$ & $19(27.9)$ \\
\hline $0-9$ & $32(25.8)$ & $14(25.0)$ & $25(36.8)$ \\
\hline $10-19$ & $47(37.9)$ & $23(41.1)$ & $10(14.7)$ \\
\hline$\geq 20$ & & & $15(22.1)$ \\
\hline Full-time GPs in practice & $15(12.1)$ & $5(8.9)$ & $15(22.1)$ \\
\hline 1 & $26(21.0)$ & $11(19.6)$ & $15(22.1)$ \\
\hline 2 & $29(23.4)$ & $14(25.0)$ & $13(19.1)$ \\
\hline 3 & $34(27.4)$ & $19(33.9)$ & $7(12.5)$ \\
\hline$\geq 5$ & $20(16.1)$ & &
\end{tabular}

to the COVID-19 pandemic, the authors were not able to send the survey to the last ten GPs and response rate could have potentially been $5 \%$ higher.

This study did not use a validated questionnaire. No relevant questionnaire existed, and a rigorous validation process was not prioritised in the development phase of the trial. However, the definition of themes and items were based on the literature. ${ }^{15,31-33}$ Formulations and face validity was tested by feedback from a user panel of GPs and oncologists during piloting. ${ }^{22}$ Moreover, the authors minimised the risk of overestimating the effect of the study by reducing the number of items for between-group analysis.

The study might be diminished in power. The sample size calculations did not refer to the secondary outcomes reported in this study. Furthermore, the numerous technical problems resulted in cancellation or alternative ways of tripartite communication in $32 \%$ of the consultations planned.

Table 3 Effect of the intervention for the selected six dichotomy items on GPs involvement in different aspects of treatment and GPs satisfaction with information and agreements of roles

\begin{tabular}{|c|c|c|c|c|c|}
\hline \multirow[b]{2}{*}{ Items } & \multirow{2}{*}{$\begin{array}{l}\text { Respons- } \\
\text { es, } n\end{array}$} & \multicolumn{2}{|c|}{ Intention-to-treat } & \multicolumn{2}{|c|}{ Per-protocol } \\
\hline & & OR $(95 \% \mathrm{Cl})^{a}$ & $P$ value & OR $(95 \% \mathrm{Cl})^{\mathrm{a}}$ & $P$ value \\
\hline $\begin{array}{l}\text { As a GP, did you experience direct contact or dialogue } \\
\text { with the Department of Oncology? }\end{array}$ & 117 & $\begin{array}{l}6.95(2.96 \text { to } \\
16.35)\end{array}$ & 0.000 & $\begin{array}{c}6.09(2.64 \text { to } \\
14.03)\end{array}$ & 0.000 \\
\hline $\begin{array}{l}\text { As a GP, I have been involved in the treatment of } \\
\text { comorbidity }\end{array}$ & 124 & 1.35 (0.67 to 2.75$)$ & 0.404 & 1.26 (0.58 to 2.72$)$ & 0.560 \\
\hline $\begin{array}{l}\text { As a GP, I have been involved in handling the patient' } \\
\text { anxiety and psychological concerns }\end{array}$ & 124 & 0.44 (0.21 to 0.92$)$ & 0.029 & 0.64 (0.31 to 1.35$)$ & 0.245 \\
\hline $\begin{array}{l}\text { As a GP, I found the information from the department to } \\
\text { help me manage the physical issues }\end{array}$ & 116 & 0.88 (0.41 to 1.88$)$ & 0.739 & 1.55 (0.70 to 3.47$)$ & 0.283 \\
\hline $\begin{array}{l}\text { As a GP, how satisfied are you, with the distribution } \\
\text { of tasks and roles regarding initiatives practice could } \\
\text { initiate concerning the patient trajectory? }\end{array}$ & 117 & 3.03 (1.35 to 6.82$)$ & 0.007 & $\begin{array}{l}4.14(1.62 \text { to } \\
10.56)\end{array}$ & 0.003 \\
\hline $\begin{array}{l}\text { As a GP, how satisfied are you, with the distribution of } \\
\text { tasks and roles of who should take care of side-effects } \\
\text { and late complications? }\end{array}$ & 121 & 1.34 (0.57 to 3.20$)$ & 0.504 & 1.49 (0.60 to 3.70$)$ & 0.389 \\
\hline
\end{tabular}


Finally, it is unknown whether any spill-over effects may have improved care for the patients in the control group, leading to a smaller impact of the intervention. To minimise the spill-over risk, the authors did not inform GPs about their patients being involved in the study if enrolled in the control group before they received the questionnaire. Although their patients may have been told about the target of the study, GPs were in some way blind. However, the blinding of patients and GPs in the intervention group was impossible. Only nine of 105 GPs had >1 patient in the study, and eight had patients in both groups. Therefore, it is believed that the risk of spill-over was minimal.

The study had the advantage of being randomised. The study included patients with various cancer types, different prognosis, health problems, and needs of supportive care. However, lung and gastrointestinal cancer were in the majority. The patient heterogeneity of the study mimics the patient population in the department as well as general practice in the Region, ${ }^{34}$ which is a strength for generalisability and future implementation.

Finally, including intention-to-treat analysis reflects on everyday clinical practice and enhances external validity. ${ }^{35,36}$ The results of the intention-to-treat and per-protocol analysis showed similar results, and the authors wonder if the introduction of the concept of cooperation between sectors and the overall goal of involving the GP, independent of the completion of the video consultation, could be a substantial component.

\section{Comparison with existing literature}

The high OR for direct contact indicates that the GPs recall the video consultations and found it useful, as indicated in the process evaluation of the trial. ${ }^{26}$ During process evaluations, it was shown that the consultations succeeded in being patient-centred, could be managed within daily clinical routines, and improved the coordination of care as perceived by patients, oncologists, and GPs. ${ }^{24,26}$ In line with a review about shared care models, stating that involving GPs and improving communication between sectors can benefit cancer care, this study indicates enhanced GPs involvement and, furthermore, that the GPs were satisfied with the distribution of tasks. ${ }^{10}$

A Cochrane review on shared care models across sectors suggests that shared care may have the potential to provide long-term benefit for patients with depression and increase effectiveness of the organisation of shared care if introduced at an early stage in the disease process using information technology..$^{11}$ Moreover, the review states that shared care model should be develop for other conditions. The PSP used video consultation at an early stage in the trajectory. Introducing sharing and the use of information technology is believed to be an essential mechanism for the study effect on GPs involvement.

Improving communication between primary and secondary care can lead to an increase in GP involvement. ${ }^{37}$ Higher GP involvement calls for easy access to sufficient knowledge. ${ }^{38}$ A shift from one-way communication in summary letters to real-time dialogue may be important for improving communication and involvement. This study has shown that arranging direct contact could increase the GP's perception of involvement in the trajectory and lead to a more precise distribution of tasks and roles.

\section{Implications for research and practice}

Despite compromised participation rates and technical challenges, this study showed that communication by bringing health professionals and patients together by video was perceived as useful, and resulted in GPs feeling better informed about their role. These results are believed to have a positive impact on the recruitment of patients and health professionals for the next generation of PSP.

The partnership intervention makes a difference at the physician level and supports the theories of the mechanisms of this shared care programme. ${ }^{22}$ Assuming reliable IT solutions were available, the authors concluded that with relatively limited effort, the sharing of 15 minutes through video across sectors as part of a routine communication can help GPs in providing better care for cancer patients. ${ }^{26}$ The COVID-19 pandemic may have increased the willingness to participate in video consultations dramatically.

Future research should focus on the need for a validated questionnaire to measure GPs' experiences with tripartite video consultations. The questionnaire in this study could be used as a template. 
Moreover, investigations on the patient-physician relationship, when GPs declined to participate in a study like this, are welcomed.

Finally, the evaluation of patient outcomes is crucial before implementation into routine care. Patient evaluation of coordination of care and health-related quality of life are among planned outcomes.

\section{Funding}

This study is financially supported by the Danish Cancer Society (reference number: R156-A1001816-S49), the Region of Southern Denmark (reference number: 14/39009), ML Jørgensen and Gunnar Hansen Foundation, and the University of Southern Denmark. The sponsors were neither involved in the planning, nor design, nor carrying out of the study.

\section{Ethical approval}

The Regional Ethics Committee on Biomedical Research in Denmark evaluated the project (reference number: S-20142000-138). They concluded that the intervention is an administrative procedure and therefore does not need approval as a biomedical trial. The Danish Data Protection Agency has approved the study (reference number: 2014-41-3534). Full research procedures are registered on www.clinicaltrials.gov (reference number: NCT02716168).

\section{Provenance}

Freely submitted; externally peer reviewed

\section{Acknowledgements}

The authors are grateful for the willingness of GPs in the Region of Southern Denmark and oncologists at the Department of Oncology, Lillebælt University Hospital to participate in this study. Moreover, the authors would like to thank the project coordinators Jette Leonhardt (Department of Oncology, Lillebaelt University Hospital, Vejle, Denmark) and Heidi Gram (Research Unit for General Practice, Odense) for the great effort in executing the trial. The authors also wish to thank the Patient and Relatives Council at Lillebælt University Hospital, Vejle, Denmark, for their invaluable participation in the development of the interventions. Finally, the authors wish to thank Soeren Raunsgaard for his extensive help with data management concerning the preparation and calculations of the data.

\section{References}

1. Grunfeld E. Primary care physicians and oncologists are players on the same team. J Clin Oncol 2008; 26(14): 2246-2247. DOI: https://doi.org/10.1200/JCO.2007.15.7081

2. Grunfeld E, Earle CC. The interface between primary and oncology specialty care: treatment through survivorship. $J$ Natl Cancer Inst Monogr 2010; 2010(40): 25-30. DOI: https://doi.org/10.1093/jncimonographs/lgq002

3. Lawrence RA, McLoone JK, Wakefield CE, Cohn RJ. Primary care physicians' perspectives of their role in cancer care: a systematic review. J Gen Intern Med 2016; 31(10): 1222-1236. DOI: https://doi.org/10.1007/s11606-0163746-7

4. Klabunde $\mathrm{CN}$, Ambs A, Keating NL, et al. The role of primary care physicians in cancer care. J Gen Intern Med 2009; 24(9): 1029-1036. DOI: https://doi.org/10.1007/s11606-009-1058-x

5. Easley J, Miedema B, O'Brien MA, et al. The role of family physicians in cancer care: perspectives of primary and specialty care providers. Curr Oncol 2017; 24(2): 75-80. DOI: https://doi.org/10.3747/co.24.3447

6. Kendall M, Boyd K, Campbell C, et al. How do people with cancer wish to be cared for in primary care? Serial discussion groups of patients and carers. Fam Pract 2006; 23(6): 644-650. DOI: https://doi.org/10.1093/fampra/ $\mathrm{cml035}$

7. Mikkelsen T, Sondergaard J, Sokolowski I, et al. Cancer survivors' rehabilitation needs in a primary health care context. Fam Pract 2009; 26(3): 221-230. DOI: https://doi.org/10.1093/fampra/cmp004

8. Hall S, Gray N, Browne S, et al. A qualitative exploration of the role of primary care in supporting colorectal cancer patients. Support Care Cancer 2012; 20(12): 3071-3078. DOI: https://doi.org/10.1007/s00520-012-1434-7

9. Barry MJ, Edgman-Levitan S. Shared decision making - pinnacle of patient-centered care. N Engl J Med 2012; 366(9): 780-781. DOI: https://doi.org/10.1056/NEJMp1109283

10. Zhao Y, Brettle A, Qiu L. The effectiveness of shared care in cancer survivors-a systematic review. Int J Integr Care 2018; 18(4): 2. DOI: https://doi.org/10.5334/ijic.3954

11. Smith SM, Cousins G, Clyne B, et al. Shared care across the interface between primary and specialty care in management of long term conditions. Cochrane Database Syst Rev 2017; 2(2): CD004910. DOI: https://doi.org/10. 1002/14651858.CD004910.pub3 
12. Schütze $H$, Chin $M$, Weller $D$, Harris MF. Patient, general practitioner and oncologist views regarding long-term cancer shared care. Fam Pract 2018; 35(3): 323-329. DOI: https://doi.org/10.1093/fampra/cmx105

13. Duggal R, Brindle I, Bagenal J. Digital healthcare: regulating the revolution. BMJ 2018; 360: k6. DOI: https://doi. org/10.1136/bmj.k6

14. Marshall $\mathrm{M}$, Shah $\mathrm{R}$, Stokes-Lampard $\mathrm{H}$. Online consulting in general practice: making the move from disruptive innovation to mainstream service. BMJ 2018; 360: k1195. DOI: https://doi.org/10.1136/bmj.k1195

15. Sabesan S, Allen DT, Caldwell P, et al. Practical aspects of telehealth: establishing telehealth in an institution. Intern Med J 2014; 44(2): 202-205. DOI: https://doi.org/10.1111/imj.12339

16. Kitamura C, Zurawel-Balaura L, Wong RKS. How effective is video consultation in clinical oncology? A systematic review. Curr Oncol 2010; 17(3): 17-27. DOI: https://doi.org/10.3747/co.v17i3.513

17. Allen DT, Caldwell P, Komesaroff PA, et al. Practical aspects of telehealth: set-up and preparation for video consultations. Intern Med J 2013; 43(10): 1133-1136. DOI: https://doi.org/10.1111/imj.12264

18. Fleissig A, Jenkins V, Catt S, Fallowfield L. Multidisciplinary teams in cancer care: are they effective in the UK? Lancet Oncol 2006; 7(11): 935-943. DOI: https://doi.org/10.1016/S1470-2045(06)70940-8

19. Pype $P$, Mertens F, Belche J, et al. Experiences of hospital-based multidisciplinary team meetings in oncology: an interview study among participating general practitioners. Eur J Gen Pract 2017; 23(1): 156-164. DOI: https://doi. org/10.1080/13814788.2017.1323081

20. Chaillou D, Mortuaire G, Deken-Delannoy V, et al. Presence in head and neck cancer multidisciplinary team meeting: the patient's experience and satisfaction. Eur Ann Otorhinolaryngol Head Neck Dis 2019; 136(2): 75-82. DOI: https://doi.org/10.1016/j.anorl.2018.10.003

21. Myhre A, Agai M, Dundas I, Feragen KB. "All eyes on me": a qualitative study of parent and patient experiences of multidisciplinary care in craniofacial conditions. Cleft Palate Craniofac J 2019; 56(9): 1187-1194. DOI: https://doi. org/10.1177/1055665619842730

22. Trabjerg TB, Jensen LH, Søndergaard J, et al. Improving continuity by bringing the cancer patient, general practitioner and oncologist together in a shared video-based consultation - protocol for a randomised controlled trial. BMC Fam Pract 2019; 20(1): 86. DOI: https://doi.org/10.1186/s12875-019-0978-8

23. Moher D, Hopewell S, Schulz KF, et al. Consort 2010 explanation and elaboration: updated guidelines for reporting parallel group randomised trials. J Clin Epidemiol 2010; 63(8): e1-e37. DOI: https://doi.org/10.1016/j. jclinepi.2010.03.004

24. Trabjerg TB, Jensen LH, Sondergaard J, et al. Investigating whether shared video-based consultations with patients, oncologists, and GPs can benefit patient-centred cancer care: a qualitative study. BJGP Open 2020; 4(2): bjgpopen20X101023. DOI: https://doi.org/10.3399/bjgpopen20X101023

25. Harris PA, Taylor R, Thielke R, et al. Research electronic data capture (REDCap) - a metadata-driven methodology and workflow process for providing translational research informatics support. J Biomed Inform 2009; 42(2): 377-381. DOI: https://doi.org/10.1016/j.jbi.2008.08.010

26. Trabjerg TB, Jensen LH, Søndergaard J, et al. Cross-sectoral video consultations in cancer care: perspectives of cancer patients, oncologists and general practitioners. Support Care Cancer 2021; 29(1): 107-116. DOI: https:// doi.org/10.1007/s00520-020-05467-0

27. Creavin ST, Creavin AL, Mallen CD. Do GPs respond to postal questionnaire surveys? A comprehensive review of primary care literature. Fam Pract 2011; 28(4): 461-467. DOI: https://doi.org/10.1093/fampra/cmr001

28. VanGeest JB, Johnson TP, Welch VL. Methodologies for improving response rates in surveys of physicians: a systematic review. Eval Health Prof 2007; 30(4): 303-321. DOI: https://doi.org/10.1177/0163278707307899

29. Pit SW, Vo T, Pyakurel S. The effectiveness of recruitment strategies on general practitioner's survey response rates — a systematic review. BMC Med Res Methodol 2014; 14: 76. DOI: https://doi.org/10.1186/1471-2288-14-76

30. Pearl A, Wright $S$, Gamble G, et al. Randomised trials in general practice - a New Zealand experience in recruitment. N Z Med J 2003; 116(1186): U681.

31. Silverman J, Kurtz S, Draper J. Skills for communicating with patients. 3rd edn. Boca Raton, FL: CRC Press; 2013.

32. Holm LV, Hansen DG, Johansen C, et al. Participation in cancer rehabilitation and unmet needs: a populationbased cohort study. Support Care Cancer 2012; 20(11): 2913-2924. DOI: https://doi.org/10.1007/s00520-012$1420-0$

33. Australian College of Rural and Remote Medicine. Handbook for the TeleHealth online education module. 2013; http://www.ehealth.acrrm.org.au/sites/default/files/content/ACRRM\%20Handbook\%20for\%20telehealth\% 20 online\%20education\%20module.pdf (accessed 11 Feb 2021).

34. Pedersen KM, Andersen JS, Søndergaard J. General practice and primary health care in Denmark. J Am Board Fam Med 2012; 25(Suppl 1): S34-S38. DOI: https://doi.org/10.3122/jabfm.2012.02.110216

35. Rothwell PM. External validity of randomised controlled trials: "to whom do the results of this trial apply?". Lancet 2005; 365(9453): 82-93. DOI: https://doi.org/10.1016/S0140-6736(04)17670-8

36. Gupta SK. Intention-to-treat concept: a review. Perspect Clin Res 2011; 2(3): 109-112. DOI: https://doi.org/10. 4103/2229-3485.83221

37. Meiklejohn JA, Mimery A, Martin JH, et al. The role of the GP in follow-up cancer care: a systematic literature review. J Cancer Surviv 2016; 10(6): 990-1011. DOI: https://doi.org/10.1007/s11764-016-0545-4

38. Nugteren IC, Duineveld LAM, Wieldraaijer T, et al. Need for general practitioner involvement and eHealth in colon cancer survivorship care: patients' perspectives. Fam Pract 2017; 34(4): 473-478. DOI: https://doi.org/10.1093/ fampra/cmw139 